\title{
Detailed expression profile of the six Glypicans and their modifying enzyme, Notum during chick limb and feather development
}

Article

Accepted Version

Creative Commons: Attribution-Noncommercial-No Derivative Works 4.0

Saad, K., Theis, S., Otto, A., Luke, G. and Patel, K. (2017) Detailed expression profile of the six Glypicans and their modifying enzyme, Notum during chick limb and feather development. Gene, 610. pp. 71-79. ISSN 0378-1119 doi: https://doi.org/10.1016/j.gene.2017.02.012 Available at https://centaur.reading.ac.uk/69037/

It is advisable to refer to the publisher's version if you intend to cite from the work. See Guidance on citing.

To link to this article DOI: http://dx.doi.org/10.1016/j.gene.2017.02.012

Publisher: Elsevier

All outputs in CentAUR are protected by Intellectual Property Rights law, including copyright law. Copyright and IPR is retained by the creators or other copyright holders. Terms and conditions for use of this material are defined in the End User Agreement. 


\section{CentAUR}

Central Archive at the University of Reading

Reading's research outputs online 
Title

Detailed expression profile of the six Glypicans and their modifying enzyme, Notum during chick limb and feather development

\section{Authors}

Kawakeb Saad ${ }^{1}$, Susanne Theis ${ }^{1}$, Anthony Otto ${ }^{1}$, Graham Luke ${ }^{1}$, Ketan Patel ${ }^{1,2, \star}$

\section{Address}

1 School of Biological Sciences, University of Reading, Reading, UK

2 Freiburg Institute for Advanced Studies (FRIAS), University of Freiburg,

Freiburg, Germany

Corresponding author ketan.patel@reading.ac.uk

\section{Address}

School Biological Sciences

University of Reading

Hopkins Building

Reading

RG6 6UB, UK 


\section{Abstract}

The development of vertebrate appendages, especially the limb and feather buds are orchestrated by numerous secreted signalling molecules including Sonic Hedgehog, Bone Morphogenetic Proteins, Fibroblast Growth Factors and Wnts. These proteins coordinate the growth and patterning of ectodermal and mesenchymal cells. The influence of signalling molecules is affected over large distances by their concentration (morphogen activity) but also at local levels by the presence of proteins that either attenuate or promote their activity. Glypicans are cell surface molecules that regulate the activity of the major secreted signalling molecules expressed in the limb and feather bud.

Here we investigated the expression of all Glypicans during chick limb and feather development. In addition we profiled the expression of Notum, an enzyme that regulates Glypican activity. We show that five of the six Glypicans and Notum are expressed in a dynamic manner during the development of limbs and feathers. We also investigated the expression of key Glypicans and show that they are controlled by signalling molecules highlighting the presence of feedback loops. Lastly we show that Glypicans and Notum are expressed in a tissue specific manner in adult chicken tissues.

Our results strongly suggest that the Glypicans and Notum have many as yet undiscovered roles to play during the development of vertebrate appendages.

\section{Introduction}

The development of the vertebrate limb and feather bud have been extensively studied as model systems to gain insights into the processes that control outgrowth and 
patterning. The chick model system offers a number of key features that make it the platform of choice to investigate these processes since it is amenable to experimentation both at the tissue and molecular level and is observable over long periods of time. Classic tissue manipulations carried out in second half of the twentieth century lead to the identification of the Zone of Polarising Activity (ZPA), the Apical Ectodermal Ridge (AER) and the dorsal ectoderm as key regulators in the development of the vertebrate limb (reviewed by Tickle (Tickle, 2004)). Thereafter, the molecular factors that originate from these tissues have been identified; Shh is produced by the ZPA and controls anterior-posterior patterning, Fgfs are secreted by the AER and promote proximal-distal development whereas the dorsal ectoderm is the source of Wnt7a (reviewed by Towers and Tickle (Towers and Tickle, 2009).

Although the formation of the limb and feather bud depends on only two cell types, the epithelia and the underlying mesenchyme, a huge number of different molecules are needed to translate the information from the three key signalling centres to ensure that the correct tissues development at the correct time and place. One key feature of limb and feather development is the necessity of molecules that either attenuate or promote signalling molecule activity (e.g. secreted Frizzled related proteins, Gremlin (Bardot et al., 2004; Bovolenta et al., 2008).

The extra cellular matrix (ECM) adds another layer of complexity in directing the activity of signalling molecules that control appendage outgrowth. Indeed there is compelling evidence that without the correct ECM, signalling activities of certain secreted proteins including the Fgfs can be essentially nullified (reviewed by Kim et al (Kim et al., 2011)). Heparan Sulfate Proteoglycans (HSPG) are a key component of the ECM and act as co-recptors for many signalling cascades (Bernfield et al., 1999). There are four major families of cell-surface HSPGs: Syndecans, beteglycans, the 
CD44 family and Glypicans (Gpc). The first three have transmembrane domains enabling them to remain at the cell membrane whereas Gpcs are tethered through a GPI link (Kreuger and Kjellen, 2012; Sarrazin et al., 2011). The Gpcs are of particular interest in the context of limb and feather development as they have been implicated in regulating the activity of Shh, Bmp, Fgf and Wnt signalling (Dwivedi et al., 2013; Fico et al., 2011). Six Gpcs have been found in vertebrates and these can be subdivided into two families comprising one group with Gpc1, Gpc2, Gpc4 and Gpc6 and the other Gpc3 and Gpc5 based sequence similarity (Veugelers et al., 1999). Furthermore two Gpcs, Division abnormally delayed (Dally) and Dally-like protein (DIp) are found in Drosophila where they have been implicated in shaping morphogen gradients during wing development by regulating wingless and Dpp activity (Drosophila homologues of Wnt and Bmps respectively (Wu et al., 2010; Yan and Lin, 2009).

Signalling cascades that regulate the development of limbs are highly conserved between invertebrates and vertebrates (Capdevila and Johnson, 2000). Based on the central role played by Gpcs during Drosophila wing development we investigated the expression of all the Gpcs and their modifying enzyme Notum (Kreuger et al., 2004) Here we show that Gpc1-5 and Notum are expressed in the developing limb and feather bud. They display intriguing expression patterns which fail to segregate with any one of the main four signalling molecules (Shh, Bmps, Fgfs and Wnts). We suggest that Gpcs act to coordinate the signalling activity of multiple ligands in specific regions of these two structures to ensure the appropriate outgrowth and patterning. We also show that signalling molecules can regulate the expression of Gpcs thereby implicating the presence of feedback loops that control ligand activity. Finally we show that Gpcs and Notum are expressed in tissue specific manner in adult birds. 


\section{Method}

\section{Cloning of chick Glypicans and Notum}

cDNA was prepared from RNA extracted from whole $\mathrm{HH}-25$ chick embryos (Hamburger and Hamilton, 1951). RT-PCR was performed using the following gene specific primers.

cGpc1 F5'-3'GCGAATCTGTCCGCAAGGCTACAC

R 3'-5'CTAAGCCGTCCCCCATCACTTCAG

cGpc2 F5'-3'GGCAAAAGAAGCAGCAGAGCCTGTTAAAG

R3'-5'TCATCACCAGGTCTCCATCACACAGC

cGpc3 F5'-3'CTGCTCGAGGAGGATGGAGGAGAAGTAC

R3'-5'CTGTACCTCTCCACGACTTCTTGCCC

cGpc4 F5'-3'GCGACCACTTGAAAGTCTGCTCACAAG

R3'-5'GCTGCTTGTGATAAACCGCTACTGGG

cGpc5 F5'-3'GAAAGTTTTCCAGCTGCGTCAGCTCG

R3'-5'GGCAAGGGTTTCTTCGCTGTCTCTTG

cGpc6 F5'-3'TTCTTGCAATTCCAGGGGAACATTTGAG

R3'-5'ATCCAAACTTGTGCCAGCAGCAGTTG

cNotum F5'-3' ATGCCTTCATGGGAGCGCTGATC

R3'-5' AACTGGTCCCTGATAGTGGGGCACG

PCR products were cloned into PDRIVE vector.

\section{Preparation of embryos}

All experiments were performed on Gallus gallus domesticus chicken embryos. Fertilised eggs were purchased from Henry Stewart and Co, UK. Eggs were incubated at $38^{\circ} \mathrm{C}$ and $80 \%$ humidity. 


\section{Whole mount in-situ hybridisation}

Whole mount in situ hybridisation was performed according to Nieto et al. (1996)(Nieto et al., 1996). A minimum of 5 embryos were processed for each experimental outcome reported here.

\section{Cryosectioning}

Embryos were washed 3 times with PBS for 15 minutes remove to PFA and they were equilibrated in 5,15 and $30 \%(\mathrm{w} / \mathrm{v})$ sucrose in PBS overnight at $4^{\circ} \mathrm{C}$ before freezing in Optimum Cutting Temperature embedding media (O.C.T.) (Leica Microsystems). $30 \mu \mathrm{m}$ sections were cut using a cryostat (Bright instruments UK). Ribbons of 3-4 sections were cut sequentially and mounted per slide. Cut sections were left for an hour to dry at room temperature and then washed for 10 minutes with PBS. They were then mounted using Hydro-mount solution (National Diagnostic) and were finally left to dry overnight before photographing.

\section{Photography}

Whole mount embryos were photographed using a Nikon CoolPix camera mounted on a Nikon dissecting microscope. Processed cyro-sections were photographed using an Axiocam digital camera fitted on a Zeiss Axioscope Fluorescent microscope connected with Zeiss Axiovision computer software version 3. Images were processed using Adobe Photoshop Elements 6.

\section{Beads implantation experiments:}

BMP4 and FGF8 proteins from R and D Systems (UK) were loaded onto 150 $\mathrm{mm}$ AffiGel Blue beads (Bio Rad, UK). Beads were first placed in small Petri dish and washed in PBS for at least 1 hour to remove preservatives. 10\% Repel silane (Sigma, UK) in chloroform was used to coat all surfaces to prevent protein adherence. $200 \mu \mathrm{g} / \mathrm{ml}$ or 
$20 \mu \mathrm{g} / \mathrm{ml}$ of BMP4, and $1 \mathrm{mg} / \mathrm{ml}$ of FGF8 were incubated with beads overnight prior to implantation. Protein soaked beads were implanted into chick forelimbs at $\mathrm{HH}-20$ for 24hr. Embryos were fixed in 4\% PFA/PBT for in-situ hybridisation.

\section{Adult chicken RNA isolation}

A 2-month old adult hen was purchased from Stokes Farm, Wokingham, UK. Tissues were dissected and snap-frozen in liquid Nitrogen and thereafter homogenized in 0.5 $\mathrm{ml}$ Trizol. The total RNA was extracted and used for the Invitrogen Single Tube cDNA synthesis protocol (In-Vitrogen, UK). cDNA was thereafter used for PCR with primers previously detailed.

\section{Results}

\section{Expression of Gpc1-Gpc6 and Notum in limb buds HH18-25}

Expression of Gcp1 was detected in both the fore and limb buds as outgrowth was initiated. A clear boundary was noted between the body and the limb at $\mathrm{HH}-18$ (Fig. 1A-A1). This relationship was maintained until at least $\mathrm{HH}-22$ in both fore and hind limb (Fig. 1A2-A3). Sections through a HH-22 fore limb showed expression of Gpc1 predominantly in the limb bud mesenchyme (Fig. 1A4).

In contrast to the robust expression of Gpc1 in the developing limbs, Gpc2 was not expressed in limb structures (Fig. 1B-B3). However transverse section of $\mathrm{HH}-22$ fore limbs showed expression proximal to the limb (Fig. 1 B4). Gcp3 showed a remarkable pattern of expressing with strong expression in the fore and hind limbs and no expression in adjacent body structure (Fig. 1C-C3). Furthermore expression was relatively uniform in limb mesenchyme with a very sharp boundary between the appendage and body (Fig. 1C4). Gpc4 was expressed strongly at $\mathrm{HH}-18$ and $\mathrm{HH}-22$ (Fig. 1D-D3). Expression at HH-22 was higher in the posterior fore and hind limb 
compared to anterior regions (Fig. 1D2-D3). In the limb expression was in the subectodermal mesenchyme with no expression in the AER. Gpc5 was expressed at low levels in the fore and hind limbs between $\mathrm{HH}-18$ and $\mathrm{HH}-22$. Again a boundary of expression exists between limb (expression of Gpc5) and body (non-expressing tissue) (Fig. 1E-E3). Transverse sections revealed expression in the mesenchyme (Fig. 1 E4). Gpc6 was not expressed in the limbs at any stage examined from $\mathrm{HH}-18$ to $\mathrm{HH}-30$ (data not shown). Notum was expressed in a dynamic pattern during early limb development. Initially at $\mathrm{HH}-18$ it was found at high levels in the distal regions and relatively uniformly in proximal portions of the fore and hind limb (Fig. 1F-F1). However at $\mathrm{HH}-22$, there was very strong expression in the distal and lesser expression in the proximal regions. Transverse sections of $\mathrm{HH}-22$ fore limbs revealed strong Notum expression in the AER and dorsal sub-ectodermal mesenchyme (Fig. 1F2-F4).

At HH-25, Gpc1 expression become localised to the AER (Fig. 2A and A2). Expression was also localised to the central portion of the limb when viewed either from the dorsal or ventral perspective where it was both ectodermal and in the immediately located mesenchyme (Fig. 2A-A2). Expression of Gpc2 was noteworthy for its expression in the stylopod and zeugopod region but not in the autopod (Fig. 2B-B1). Transverse section reveal expression in the sub-ectodermal mesenchyme (Fig.2B2). The predominant Gpc2 expression in the stylopod and zeugopod was maintained at $\mathrm{HH}$ 28 (Fig. 3A and 3A1). Thereafter it was upregulated in the inter-digit region (Fig. 3A23A3). Gpc3 showed strong expression at the margins of the limb (Fig. 2C-C1). At the distal extreme, expression was under the AER (Fig. 2C2). This pattern was maintained until HH-30 (Fig. 3B-B3). Gpc4 displayed a similar expression pattern to Gpc3 and its expression in the posterior margin was in the ectoderm and adjacent mesenchyme 
(Fig. 2D-D2). Gpc5 showed a less distinct pattern compared to the previously described genes with a prominent zone in the centre when a $\mathrm{HH}-25$ limb was viewed from either the dorsal or ventral perspective (Fig. 2E-E1). Expression was in the subectodermal tissue (Fig. 2E). Notum was expressed prominently in the AER (Fig. 2FF2) as well in the central ectoderm (Fig. 2F2).

These results show that the Gpc/Notum axis was expressed in tissues at the margin of the limb at $\mathrm{HH}-25$ with no expression of any genes in the central mesenchyme.

\section{Expression of Gpc2-Gpc5 in the leg at E10}

Late expression of Gpcs and Notum was examined by profiling transcription in the leg at E10. By this stage expression of Gpc1 and Notum was not evident. On the other hand Gpc2, 4 and 5 all showed expression in the sub-mesenchymal regions immediately proximal to the phalanges (Fig. 4A-A1,C-C1 and D-D1). Weak expression of Gpc3 was identified in the tendons anlagen. Gpc5 expression was noted in the perichondrium (Fig. 4D1). Gpc3 had a distinct expression profile; localised to the distal tip of the digits in sub-mesenchymal tissue (Fig. 4B-B1).

\section{Expression of Gpc1-5 and Notum in feathers}

We examined the expression of Gpcs and Notum in feathers as the development of these structures are controlled by secreted signalling proteins including Shh, Bmps FGfs and Wnts. Expression of Gpc1 was prominently expressed in the pre-feather bud primordia (Fig. 5A) and the only gene that was expressed in the ectoderm (Fig. 5A2). Gpc1 was initially expressed with a bias towards the posterior aspect of the feather bud ectoderm but as the bud elongated it was in the distal tip albeit at a lower level (Fig. 5A1). Tissue sections revealed highest level in the posterior ectoderm, with a 
lower level in the anterior bud ectoderm and the lowest in the distal cells (Fig.5A1-A2). Gcp2 and 3 were both only expressed once budding had started (Fig. 5B and C). Expression of both genes were in the mesenchyme and noteworthy for Gpc3 was concentrated at the base of each bud (Fig. 5C2). Gpc4 and 5 were expressed in a similar manner in the feather bud mesenchyme (Fig. 5D1-D2, E1 and E2). Notably, Gpc4 was expressed in the pre feather primordia unlike Gpc5 (Fig. 5D and E). Notum was only expressed in formed feather buds (Fig. 5F) and localised to the subectodermal mesenchyme in three domains based on intensity. There was no expression in the anterior sub-ectodermal mesenchyme, some expression in the middle portion of sub-ectodermal mesenchyme and the highest level at the posterior of this tissue (Fig. 5F2).

\section{Regulation of key Gpcs by Fibroblast Growth Factor (FGF) protein}

A number of studies have shown that Gpcs regulate the activity of Fgfs (Galli et al., 2003; Lai et al., 2008; Qiao et al., 2003). However we wanted to investigate whether there were reciprocal relationships; Gpcs modulate Fgf activity and that Fgf regulate Gpc at the expression level. For this study we focused on three Gpcs which have been extensively shown to influence Fgfs signalling: Gpc1, 3 and 4 (Galli et al., 2003; Lai et al., 2008; Qiao et al., 2003). To that end we implanted beads soaked in FGF8 and implanted them into developing chick limb buds for a period of $24 \mathrm{hr}$ and then assessed expression with in-situ hybridisation. FGF8 caused a down regulation of Gpc1 and 3 (Fig. 6 A-B1). In contrast, FGF8 caused an upregulation of Gpc4 (Fig. 6 C-C2). Therefore FGF signalling has a gene specific effect on Gpc expression.

\section{Regulation of key Gpcs by Bone Morphogenetic Protein (BMP)}


Bmps have a key role to play in the development of the limb and are expressed in the limb mesenchyme in regions that overlap with the expression of Gpc1-5 (Amthor et al., 1998). We therefore assessed the influence of Bmp (BMP-4) on Gpc expression. Implantation of beads soaked in BMP4 at concentration of $0.2 \mathrm{mg} / \mathrm{ml}$ resulted in the total repression of Gpc1, 2 and 5 (Fig. 7A, \&B and 7E). Interestingly BMPs at this concentration caused the decrease of Gpc1 not only in the mesenchyme but also in the AER (Fig. 7A2). On the other hand, expression of Gpc3 and 4 was down regulated near the bead but up-regulated further away (Fig. 7C-C2 and D-D2).

The interesting observation of a distance effect on Gpc3 and 4 caused by BMP4 bead implantation led us to investigate whether concentration of the protein was a factor in the regulation of these genes. To address this issue we implanted beads at a lower concentration $(0.02 \mathrm{mg} / \mathrm{mg})$ and assayed expression. Indeed we found that lower levels of BMPs resulted in an expression in the central regions of the limb buds in areas that would otherwise express very little transcript. (Fig. 8A-B2).

Therefore these results show that two major signalling molecules regulate the expression of Gpcs during chick limb development. The data presented shows the presence of mutual regulatory cascade between FGF/BMP and Gpcs.

\section{Expression of Gpc1-6 and Notum in adult chicken tissues}

We investigated the expression of Gcp1-6 and Notum in adult female tissues using an RT-PCR approach. Our results show that expression in tissues is highly gene dependent with Gpc1, Gpc2 and Notum being expressed in essentially the same tissues and at relatively high levels (Fig. 9 and Table 1). At the other end of the spectrum, Gpc6 in keeping with its restricted expression in the developing limb, was only expressed in the kidney of the 19 surveyed tissues. Another interesting point was 
the finding that a few tissues generated two transcripts, possibly through alternative splicing (e.g. liver Gpc1,2,3 and 5) (Fig. 9). Of particular note was the finding that Notum was expressed in the bone. However we were unable to detect expression of any $G p c$ in this tissue. In contrast, the stomach expressed 4 Gpcs (1,2,4 and 5) but no Notum (Fig. 9 and Table 1).

\section{Discussion}

Epithelial-mesenchymal signalling interactions are a key feature of all vertebrate appendage outgrowth programmes. The inter dependency of both tissues ensure that the correct structures form in the correct place and time. It underpins a fundamental difference between appendage development in vertebrates and invertebrates. The former has an essential role for the mesenchyme whereas in insects (for example the wing) develops from a double-layered epithelium.

Although the development of two of the most characterised appendage programmes in vertebrates, the limb and feather bud involve only two local tissues (epithelium and mesenchyme) it is nevertheless a very complex programme. In the limb for example there are key roles played by four families of secreted proteins (Shh, Bmp, Wnt and Fgf). Often there are many members of each family to consider and specific antagonising molecules (e.g. Ptc for Shh, gremlin, follistatin and noggin for Bmps, secreted frizzled for Wnts and Sprouty for Fgfs). We consider that the complexity of the vertebrate appendage development programme will inevitably increase since we are going to have to take into account the activity of Gpcs in this process for two important reasons. Firstly this single family of proteins can modulate all the major signalling players previously mentioned and secondly there are not only five members to take into account but also their regulator, Notum. 
In the developing limb bud the Gpc/Notum show some striking features. One of the most prominent is that of Gpc3 where the gene is expressed in a limb bud specific manner highly reminiscent to the leg/wing expression of Tbx4 and 5 (RodriguezEsteban et al., 1999). Like Gpc3, Tbx4 and 5 are expressed in the mesenchyme thereby raising the possibility that $T b x 4$ and 5 through their ability to regulate transcriptions may control the expression of Gpc3 (Smith, 1997). Another fascinating profile is that of Notum with is expression solely in the dorsal sub-ectodermal mesenchyme. It is noteworthy that ectoderm over this region has been shown to express the dorsalizing secreted protein Wnt7a (Parr and McMahon, 1995). Therefore it remains a possibility that that activity of Wnt7a could be regulated by a Gpc, for example Gcp 1/3/4 (due to their presence in target tissues) and that the Gpcs activity is further refined by Notum.

Nevertheless our detailed profiling reveals one important feature of the Gpc/Notum axis in the developing limb bud which is that none of the individual genes map to the expression domain of only one key signalling molecule (Fig. 10A). This suggests that Gpcs could act to coordinate the activity of a number of signalling molecules in a particular tissues at specific stage of development. For example, Gpc3 which is mutated in Simpson-Golabi-Behmel syndrome (SGBS) and is thought to result in increased Shh activity (Capurro et al., 2008; Pilia et al., 1996) is expressed in a domain which encompasses the ZPA but extends much further into the limb (Fig. 10A). Some of the features of Golabi-Behmel syndrome (SGBS) and the mouse Gpc3 null mouse model can be attributed to over active hedgehog signalling including increases in cell proliferation, but other expected outcomes for example changes in hair patterning are not seen (Chiao et al., 2002). Likewise we suggest Gcp3 may in some regions of the 
chick limb bud regulate the activity of Shh but in other regions for example the anterior mesenchyme it may act on other pathways.

Our work also shows that signalling proteins can regulate the expression of the Gpcs in a ligand specific and ligand concentration specific manner. This is highly reminiscent to the negative feedback loops for Shh and Bmp signalling that fine tune signalling during vertebrate development (Amthor et al., 1999; Sanz-Ezquerro and Tickle, 2000). Again we suggest that combinations of secreted proteins act in a tissue specific manner to regulate the expression of Gpcs rather than at the ligand identity level.

This study shows that the Gpc/Notum axis is likely to play a role in the outgrowth of the feather buds. One result that is striking is the relatively late expression of the genes with regard to feather development. Major patterning molecules are expressed in primitive pre-bud regions which control features such as spacing (Patel et al., 1999). The temporal profile of Gpcs developed here suggests that they control subsequent growth and patterning of feather buds and not the initial events such as cell aggregation that underpin this process (Widelitz and Chuong, 1999). Similar to the situation in the limb bud, the expression of Gpcs fail to segregate with solely one signalling axis in the feather bud (Fig. 10B). Taking Gpc3 as an example due to its documented relationship with Shh, we show that the former is expressed in the base of the bud mesenchyme whereas Shh is expressed in the distal ectoderm (Widelitz et al., 1999). Gpc3 expression does overlap with the expression of the Shh receptor, Ptc which is expressed through the bud mesenchyme (McKinnell et al., 2004). Therefore Gpc3 could regulate Shh activity solely in the base of the bud. The relevance of this in a biological context remains to be determined. It is unlikely to be proliferation since this has been shown to be localised to the posterior mesenchyme ((Chen et al., 1997; 
Desbiens et al., 1992). However we do note that the high proliferation zone maps to a region with elevated Notum expression.

In summary, our work shows that the Gpcs and Notum are likely to be components that control the outgrowth and patterning of both the limb and feathers in the chick embryo (summarised in Fig. 10). It would be surprising if they were not also involved in other similar processes such as hair and genital tubercle development as these all share signalling cascades (Perriton et al., 2002; Petiot et al., 2003). In order to determine the role of the Gpcs in limb and feather development it will be necessary to identify the signalling cascades that they regulate. This aim could be achieved through a candidate approach whereby down-stream signalling molecules are investigated following the over-expression of specific Gpcs (for example monitoring changes in phosphorylation of smad1/5/8 as a readout of Bmp signalling ((Ueki and Reh, 2012). However this approach is unlikely to identify all the pathways regulated by Gpcs. We suggest a more comprehensive understanding of the role of Gpcs could be developed by firstly over-expressing or down regulating their expression followed by a mass spectroscopy profiling of all proteins. This approached could be refined by taking advantage of recent technological developments that now allow the phosphorylation of proteins to be assessed from relatively little starting material using phospho/mass spectroscopy (Dephoure et al., 2013).

\section{Acknowledgements}

We thank Dr Darius Widera for helping with figure design.

\section{Legends}




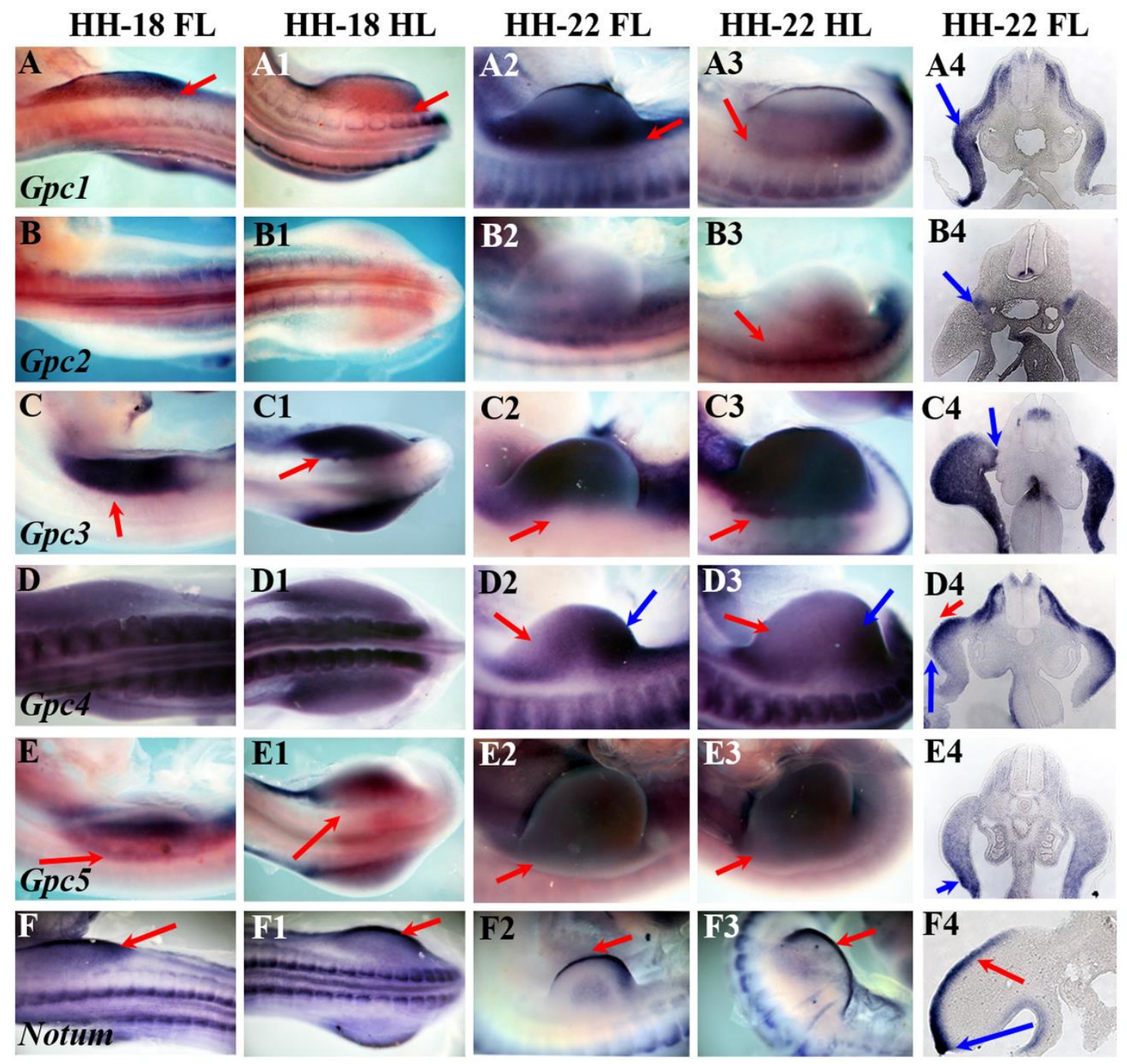

Figure 1 Expression of Gpc1-5 and Notum and HH-18 and HH-22

(A-A1) Gpc1 expression higher in limbs compared to adjacent body tissue at $\mathrm{HH} 18$ and $\mathrm{HH}-22$ (red arrows). (A4) Expression in the mesenchyme of $\mathrm{HH}-22$ fore limb (blue arrow). (B-B3) Extremely low levels of Gpc2 expression between $\mathrm{HH}-18$ and $\mathrm{HH}-22$. (B4) Expression of Gpc2 at the proximal boundary in the mesenchyme (blue arrow). (C-C3) Distinct boundary of Gpc3 expression in limb tissue compared to nonexpressing body tissue at $\mathrm{HH} 18$ to $\mathrm{HH}-22$ (red arrow). (C4) Gpc3 expression in the fore limb mesenchyme of $\mathrm{HH}-22$ embryo showing $\operatorname{limb}$ /non limb boundary (blue arrow). (D-D1) Uniform expression of Gpc4 at HH-18 in limbs. (D2-D3) Stronger Gpc4 expression in the posterior (blue arrow) compared to anterior regions (red arrow). (D4) 
Gpc4 expression in the fore limb mesenchyme of $\mathrm{HH}-22$ embryo (red arrow). Note no expression in the AER ((blue arrow). (E-E3) Boundary of Gpc5 expression in limb tissue compared to non-expressing body tissue at $\mathrm{HH} 18$ to $\mathrm{HH}-22$ (red arrow). (E4) Gpc5 expression in the fore limb mesenchyme of $\mathrm{HH}-22$ embryo with greater levels in ventral regions (blue arrow) compared to dorsal. (F-F3) High levels of Notum expression in distal regions of fore and hind limb at $\mathrm{HH} 18$ and $\mathrm{HH}-22$ (red arrows). (F4) Expression of Notum in the AER (blue arrow) and dorsal sub ectodermal mesenchyme (red arrow).

Figure 2 Expression of Gpc1-Gpc5 and Notum at HH-25 


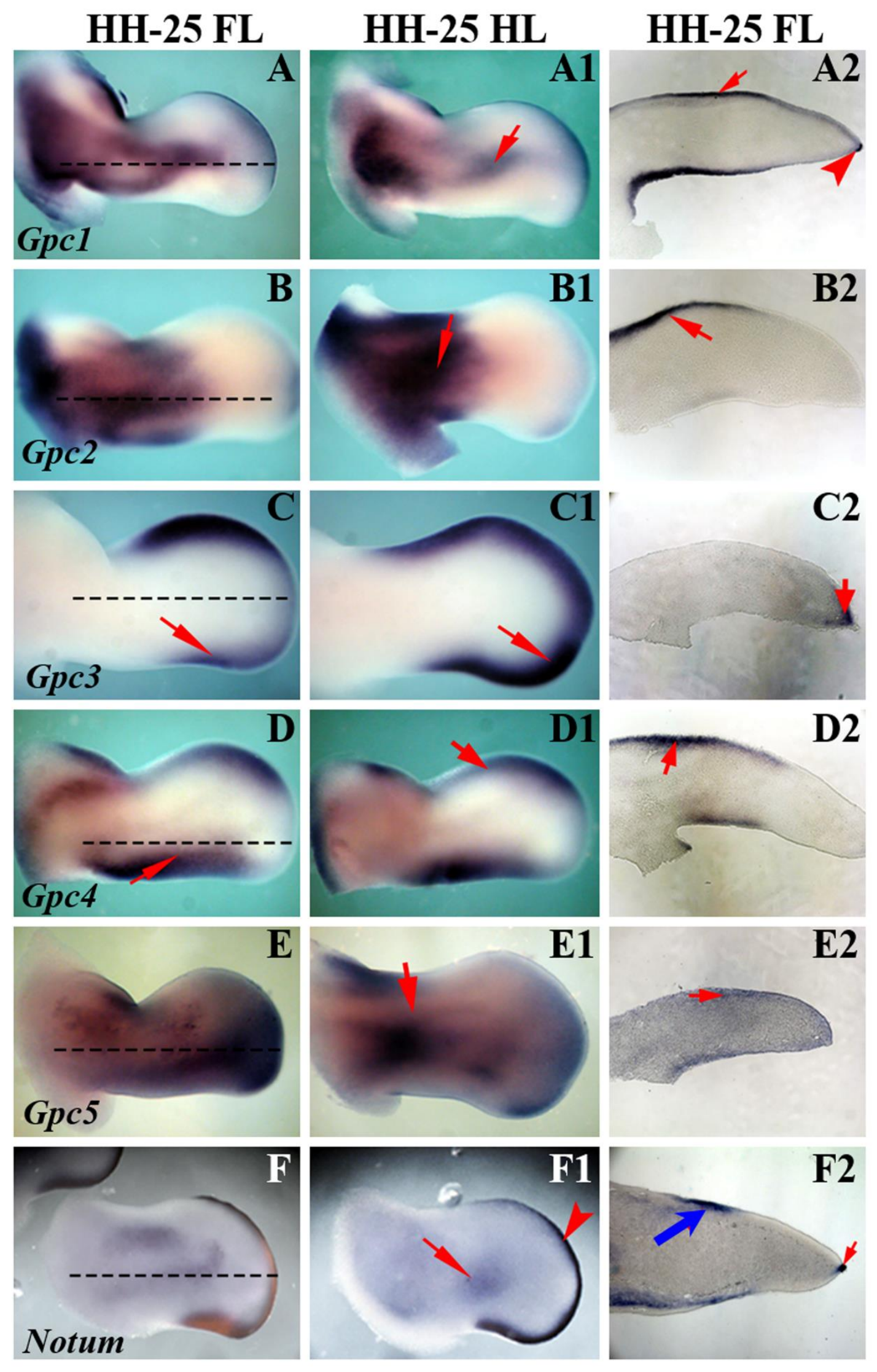


Broken line indicates section plane for images A2-F2. (A-A2) Gpc1 expression. Expression was prominent in the AER (A and arrowhead A2). (A2) Expression in central domain (red arrow) localised to the ectoderm and at lower level in adjacent mesenchyme (A2 arrowhead). (B-B2) Expression of Gpc2 at HH-25. Expression in stylopod and zeugopod (B-B1 red arrow). (B2) Expression localised to sub-ectodermal mesenchyme (red arrow).

(C-C2) Gpc3 expression at the peripheral aspects of the limb bud. (C) Expression on dorsal side was stronger at anterior compared to posterior (red arrow). (C1) On ventral side expression was more prominent on posterior side (red arrow). (C2) expression of Gpc3 localised to sub AER region (red arrow). (D-D2) Expression of Gpc4. (D) Expression in the posterior (red arrow) and (D1) anterior margin (red arrow). (D2) Sections reveal expression in the ectoderm and adjacent mesenchyme (red arrow). (EE2) Expression of Gpc4. (D-D1) Expression at the posterior and anterior margin (red arrows). (D2) Expression in ectoderm and sub-ectodermal mesenchyme. (E-E2) Expression of Gpc5. (E) Diffuse dorsal expression. (E1) Expression in central ventral region of limb (red arrow). (E2) Expression in sub-ectodermal region red arrow). (FF2) Expression of Notum. (F-F1) Expression at low level in central region of limb (red arrow) and at distal margin (red arrowhead). (F2) Notum expression in the AER (small red arrow) and ectoderm (large blue arrow).

Figure 3 Expression of Gpc2 and Gpc3 at HH-28 and $\mathrm{HH}-30$ 

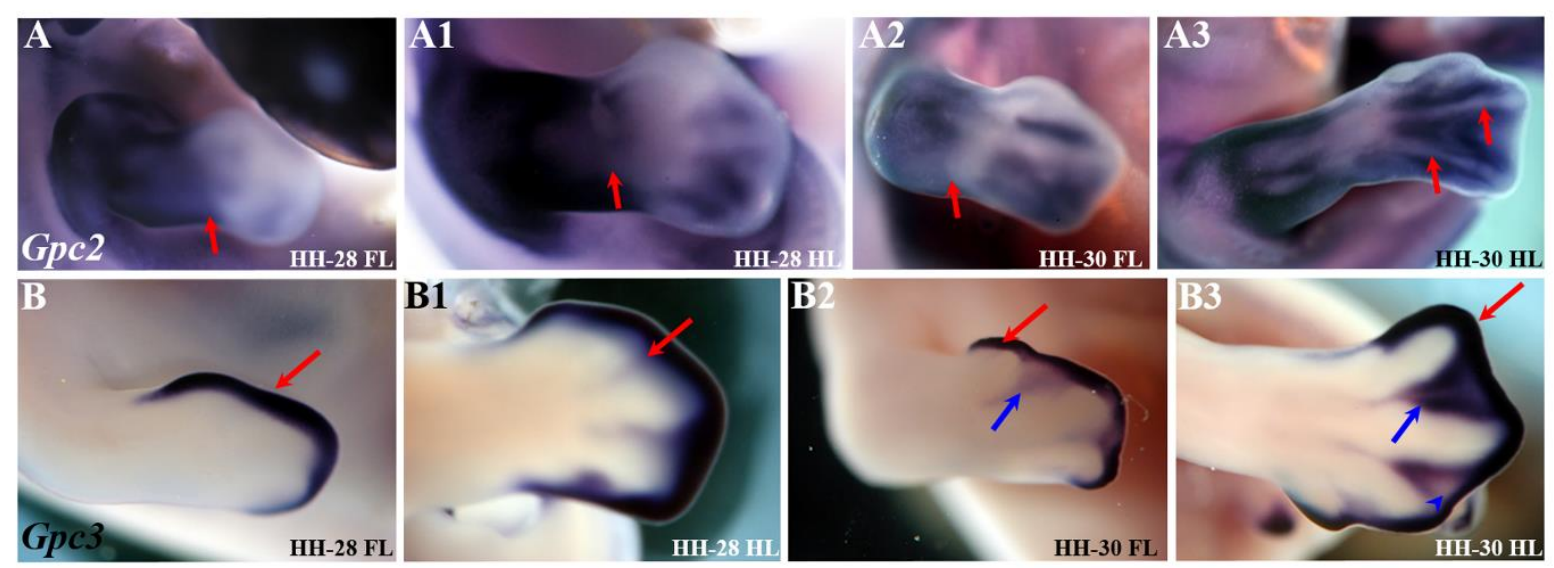

(A-A3) Expression of Gpc2. (A-A1) Gpc2 expression showing boundary between zeugopod and autopod (red arrow)/ (A2) Expression of Gpc2 at HH-30 in fore limb still has boundary at the edge of zeugopod domain (red arrow). (A3) Expression upregulated in regions adjacent to condensing digits (red arrows). (B-B3) Expression of Gpc3. (B) Expression maintained at distal limb margin (red arrow). (B1) Expression up-regulated in inter digit region of hind limb at HH-28 (red arrow). (B2) Expression of Gpc3 at high regions in distal regions (red arrow) with low levels in inter digit fore limb at $\mathrm{HH}-30$, blue arrow). (B3) Expression of Gpc3 at high regions in distal regions (red arrow) with low levels in inter digit fore limb at $\mathrm{HH}-30$ (blue arrow) and regions in inter digits showing no expression (blue arrowhead).

Figure 4 Expression of Gpc2-Gpc5 in leg at E10 

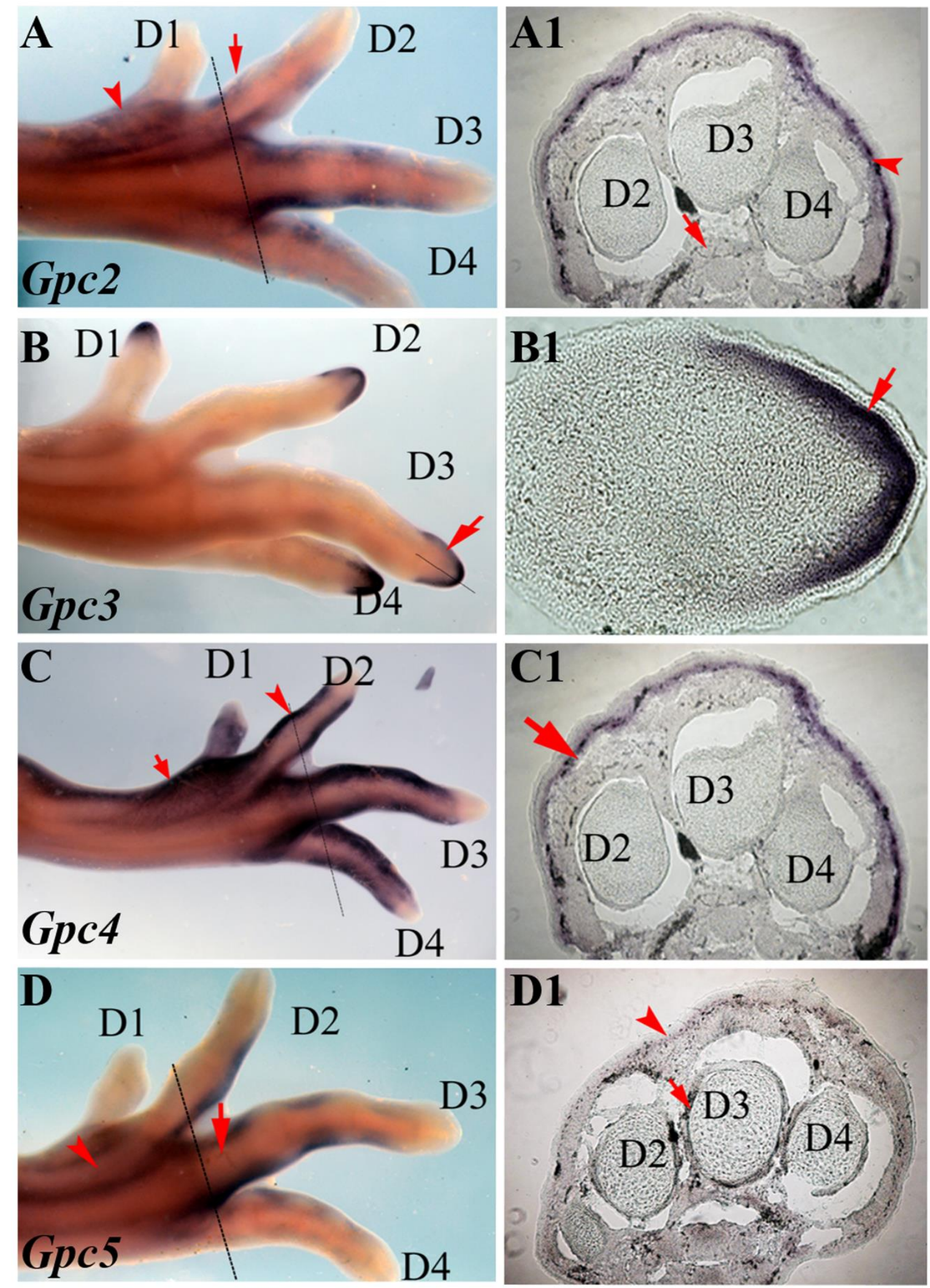

(A-F) Dorsal view of the leg at E10 for Gpc2-Gpc5 expression. (A'- D') Transverse sections at plane indicated by dotted line. (A) Gpc2 expression lateral sides of each 
digit (red arrows) and in proximal overlying tissue (red arrowhead). (A1) Expression of Gpc2 in sub-ectodermal mesenchyme (red arrowhead) and low levels adjacent to tendons (red arrow). (B) Gpc3 expression at tips of digits (red arrow). (B1) Gpc3 expression in sub-ectodermal mesenchyme (red arrowhead). (C) Gpc4 expression around digits (red arrowhead) and in more proximal region of the leg (red arrow). (C1) Expression of Gpc4 in sub-ectodermal mesenchyme. (D) Gpc5 expression round phalanges (red arrow) and metatarsals (red arrowhead). (D1) Low levels of Gpc5 expression in sub-ectodermal mesenchyme (red arrowhead) and perichondrium (red arrow).

Figure 5 Expression of Gpc1-Gpc5 and Notum in feathers 

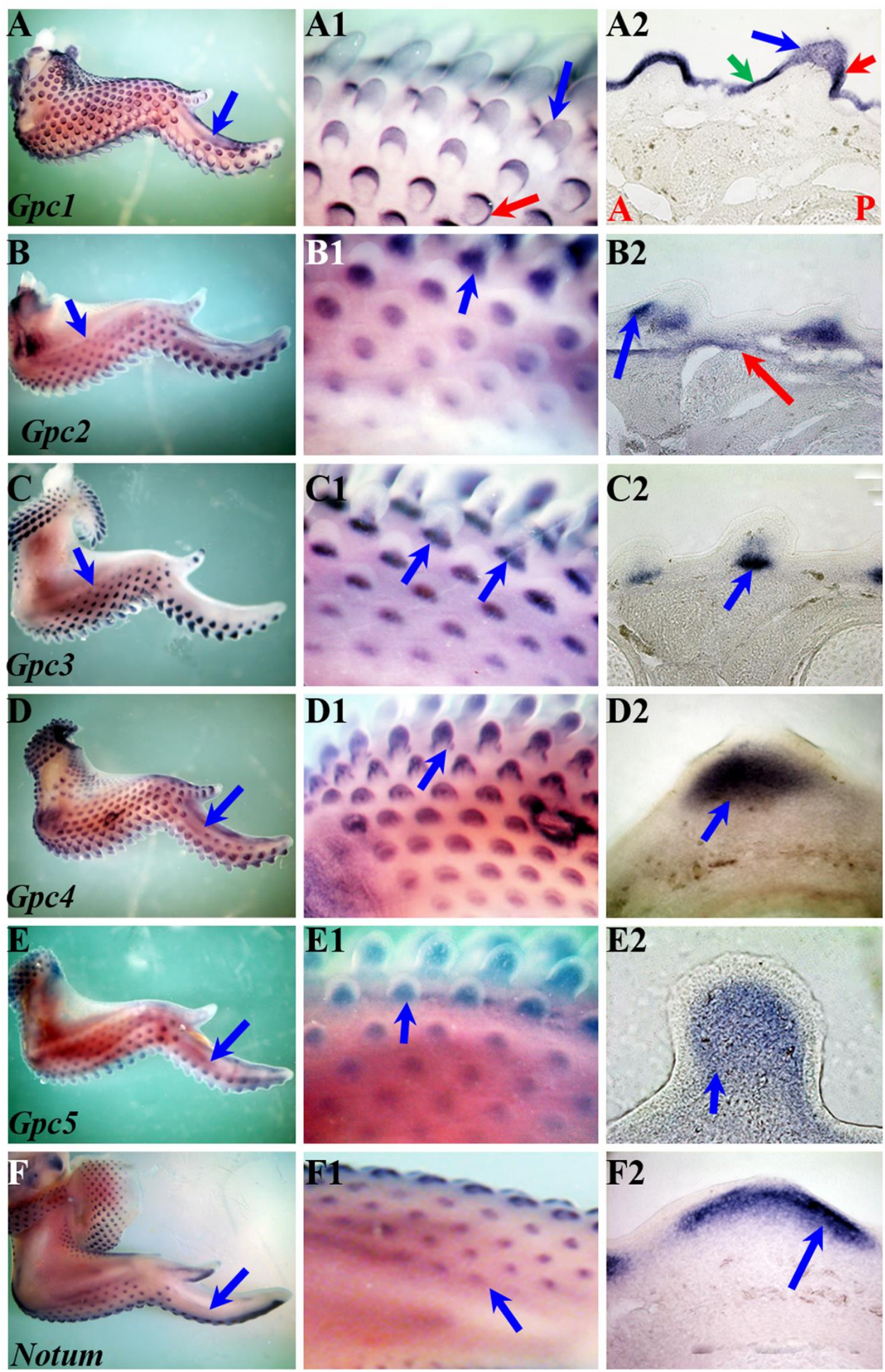
(A-A2) Gpc1 expression (A) Gcp1 was expressed in both developed feather buds as well as in the pre-feather bud primordia (blue arrow). (A1) high power image showing Gpc1 expression with a bias towards the posterior aspect of young feather buds (red arrow). In more developed buds expression in distal regions (blue arrow). (A2) Expression at high level in posterior bud ectoderm (red arrow), lower level in anterior ectoderm (green arrow) and lowest in distal ectoderm (blue arrow). (B-B2) Gpc2 expression. (B) Expression initiated with development of buds (blue arrow). (B1) Expression in mesenchyme (blue arrow). (B") Mesenchymal expression predominantly in the proximal portion of the bud (blue arrow). Low level of expression in deeper dermis (red arrow). (C-C2) Expression of Gcp3. (C) Expression initiated in developed buds (blue arrow). (C1) Expression at base of bud (blue arrows). (C2) Expression in mesenchyme at proximal aspect of feather bud (blue arrow). (D-D2) Expression of Gpc4. (D) Low levels of Gpc4 in pre-bud primordia (blue arrow). (D1) Expression in centre of feather bud (blue arrow). (D2) Expression in mesenchyme approaching the ectoderm (blue arrow). (E-E2) Gpc5 expression. (E) Weak expression in pre feather bud track (blue arrow). (E1) Inner feather bud expression evident by whole mount (blue arrow). (E2) Mesenchymal expression in feather bud (blue arrow). (F-F2) Notum expression. (F) Expression is absent in pre-feather buds tissue (blue arrow). (F1) Expression in feather buds (blue arrow). (F2) Expression in subectodermal mesenchyme with a bias for the posterior (blue arrow). Note the absence of expression in anterior sub-ectodermal mesenchyme (red arrow).

Anterior- Posterior axis indicated by red ' $\mathrm{A}$ ' and ' $\mathrm{P}$ ' respectively in A2 and same in B2F2.

Figure 6 Regulation of Gpc expression by FGF10 
Control

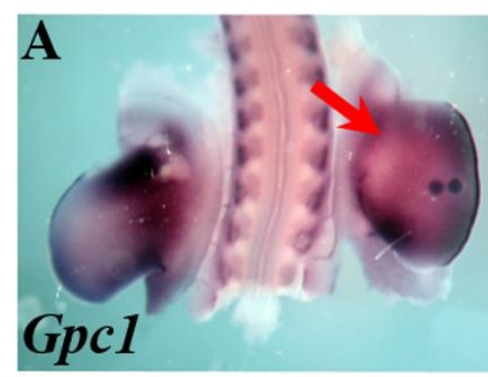

B

\section{Gpc4}
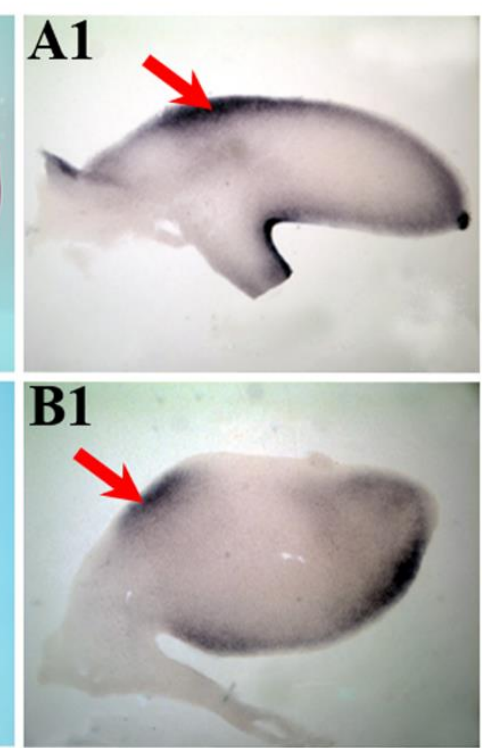

C1

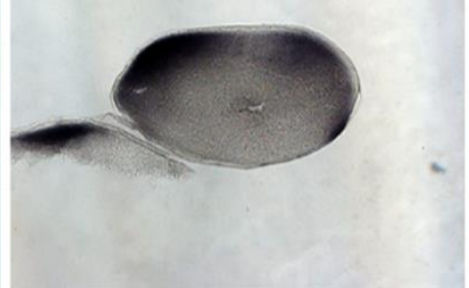

C2

FGF

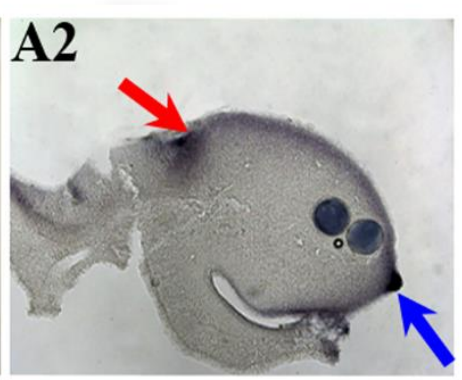

B2
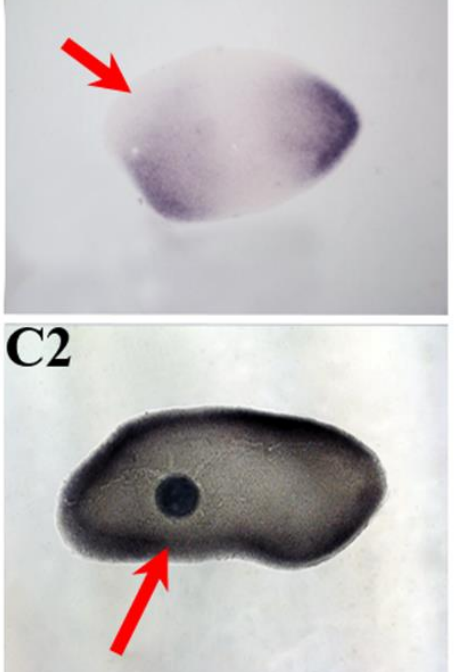

(A-A2) Expression of Gpc1. (A) Decrease in Gpc1 expression in anterior domain (red arrow) following implantation of FGF10 beads. (A1) High level of Gpc1 expression in dorsal proximal mesenchyme in control side (red arrow). (A2) FGF10 beads (blue spheres) cause a down regulation of Gpc1 (red arrows). Note expression in the AER was maintained (blue arrow). (A-B2) Expression of Gpc3. (B) Decrease in anterior and posterior domains of Gpc3 viewed in whole mount. (B1) High levels of Gpc3 in anterior domain in control limb (red arrow). (B2 (decrease in Gpc3 expression in anterior domain (red arrow). (C-C2). Expression of Gpc4. (C) up regulation of Gpc4 expression following FGF10 bead implantation in anterior (red arrowhead) and posterior (red arrow) domains. (C1) Control limb showing predominant dorsal Gpc4 expression (C2) Operated side showing Gpc4 expression in ventral limb mesenchyme (red arrow). 
Figure 7 Regulation of Gpc expression by high concentration BMP-4 $(0.2 \mathrm{mg} / \mathrm{ml})$ 
Control
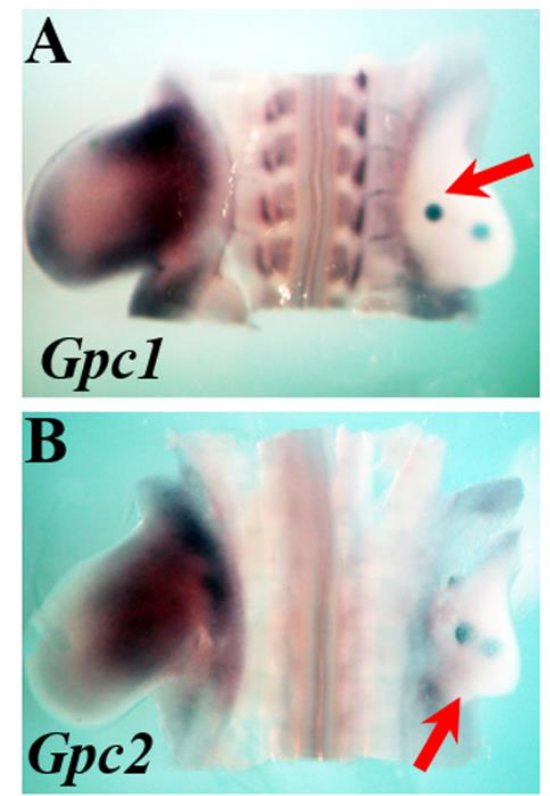

C

Gpc3

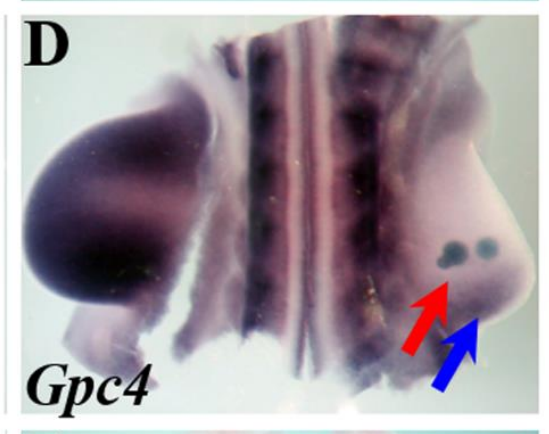

E

Gpc5
A1

B1

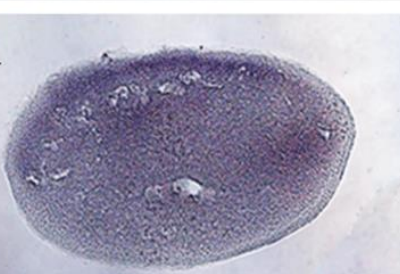

C1

D1

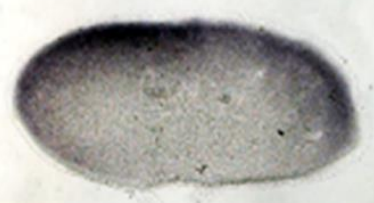

E1

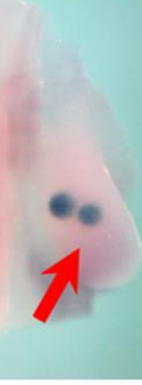

\section{BMP High}

A2

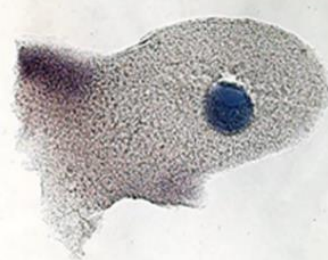

B2

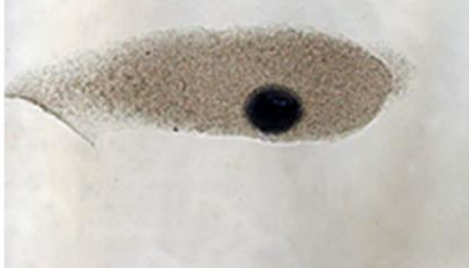

C2

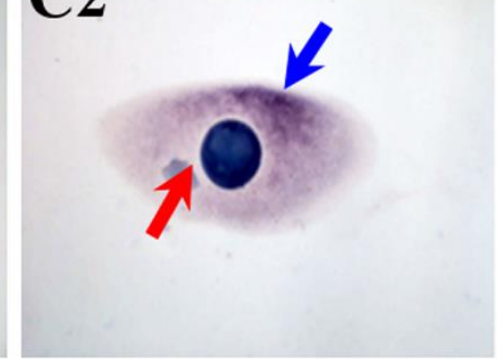

D2

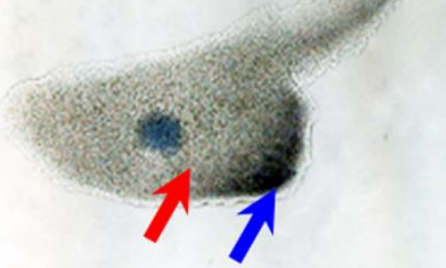

E2

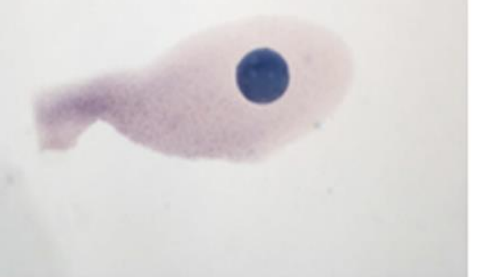

(A-A2) Expression of Gpc1. (A) Expression of Gpc1 downregulated throughout limb mesenchyme (red arrow). (A1) Expression in mesenchyme and AER (red arrow). (A2) 
Expression in decreased in the mesenchyme and AER (red arrow). (B-B2) Expression of Gpc2. (B) Expression of Gpc2 downregulated throughout limb mesenchyme (red arrow). (B1) Expression in mesenchyme. (B2) Expression in decreased in the mesenchyme. (C-C2) Expression of Gpc3. (C) Expression of Gpc3 maintained in some portions of limb (blue arrow). (C1) Expression of Gpc3 in mesenchyme. (C2) Gpc3 expression in decreased in the mesenchyme near the bead (red arrow) but maintained further away (blue arrow). (D-DC2) Expression of Gpc4. (D) Expression of Gpc4 decreased near the beads (red arrow) but present further away (blue arrow). (D1) Expression of Gpc4 in mesenchyme. (D2) Gpc4 expression in decreased in the mesenchyme near the bead (red arrow) but maintained further away (blue arrow). (EE2) Expression of Gpc5. (E) Expression of Gpc5 downregulated throughout limb mesenchyme (red arrow). (E1) Gpc5 expression in mesenchyme. (E2) Gpc5 expression in decreased by BMP4 in the mesenchyme.

Figure 8 Regulation of Gpc expression by low concentration BMP-4 $(0.02 \mathrm{mg} / \mathrm{ml})$ 
Control

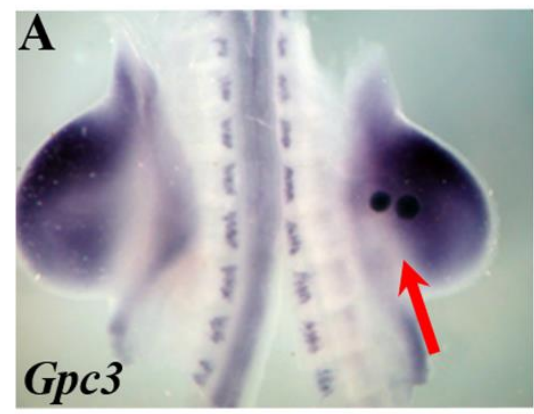

B

\section{Gpc4}

A1

B1

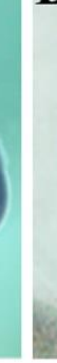

BMP Low

A2

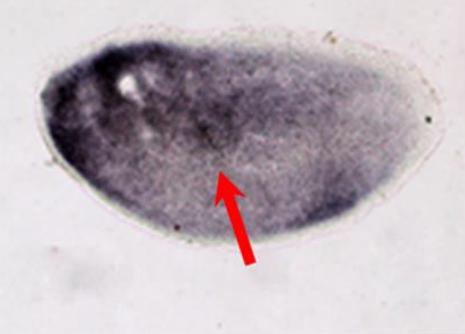

B2

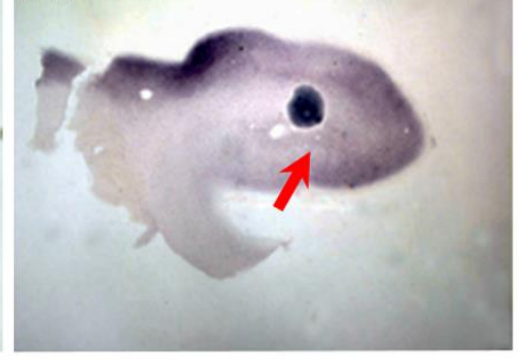

(A-A2) Expression of Gpc3. (A) Expression of Gpc3 extends to bead (red arrow). (A1)

Expression of Gpc3 in mesenchyme in control limb. (A2) Gpc3 expression in increased in centre of limb (red arrow). (B-B2) Expression of Gpc4. (B) Expression of Gpc4 up regulated in anterior and posterior (blue arrow) domains (red arrow). (B1) Expression of Gpc4 in mesenchyme in control limb. (B2) Gpc4 expression in increased in centre of limb (red arrow).

Figure 9 Expression of Gpc and Notum in adult female chicken tissues 


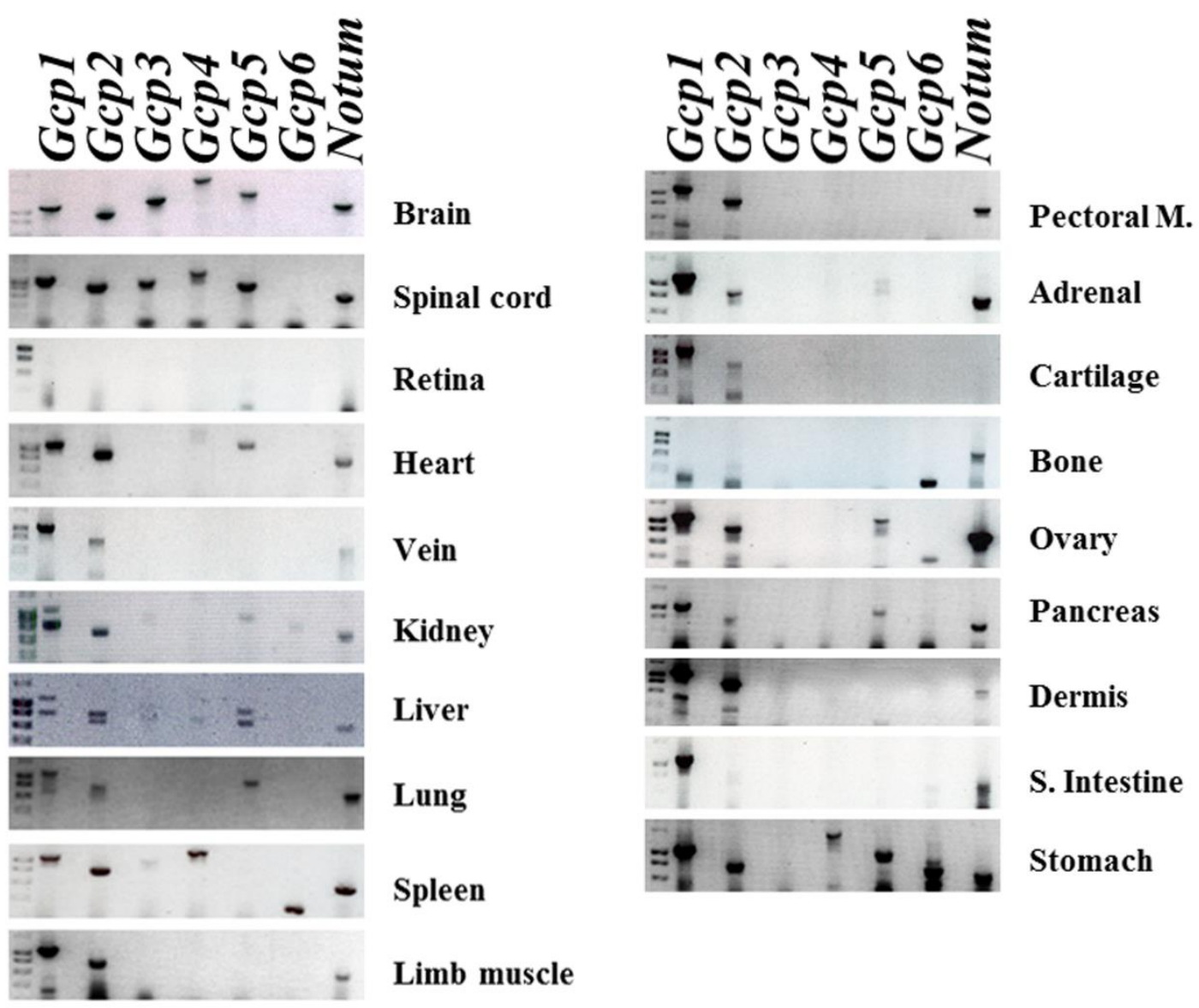

RT-PCR of 19 hen tissues assessed by gel electrophoresis.

Figure 10 Schematic of Gpc/Notum expression in chick limb and feather bud 


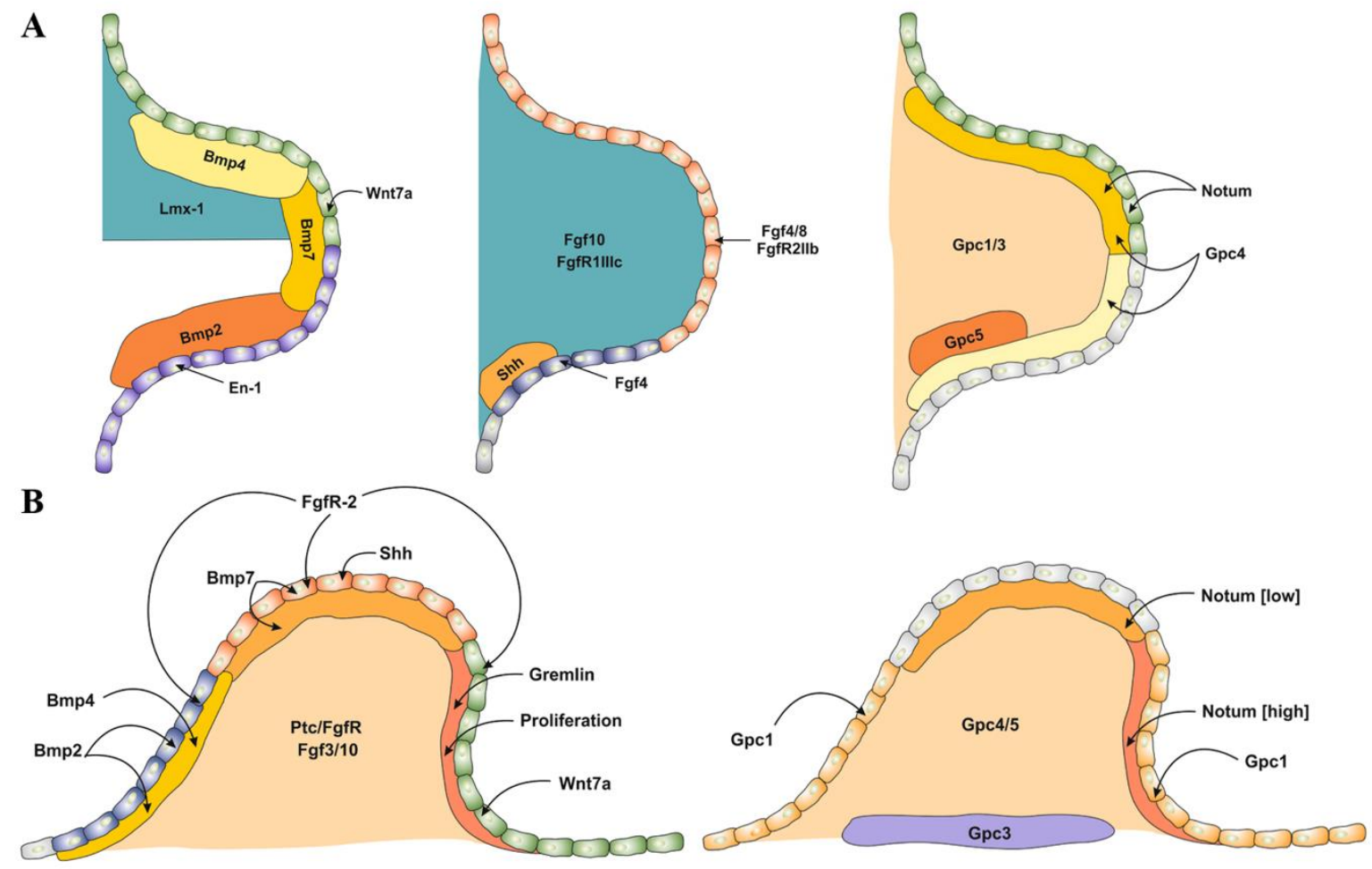

(A) Expression domains of key signalling molecules and Gpcs/Notum in the chick limb bud. (B) Expression domains of key signalling molecules and Gpcs/Notum in the chick feather bud. 
Table 1

Tabulation of Gpc and Notum expression in adult female chicken tissues

$\mathrm{XX}$ indicates high expression, $\mathrm{X}$ indicates presence of lower level of transcripts and (S) more than one transcript.

\begin{tabular}{|l|c|c|c|c|c|c|c|}
\hline & Gpc1 & Gpc2 & Gpc3 & Gpc4 & Gpc5 & Gpc6 & Notum \\
\hline Brain & $\mathrm{XX}$ & $\mathrm{XX}$ & $\mathrm{XX}$ & $\mathrm{XX}$ & $\mathrm{XX}$ & & $\mathrm{XX}$ \\
\hline Spinal cord & $\mathrm{XX}$ & $\mathrm{XX}$ & $\mathrm{XX}$ & $\mathrm{XX}$ & $\mathrm{XX}$ & & $\mathrm{XX}$ \\
\hline Retina & & & & & & & \\
\hline Heart & $\mathrm{XX}$ & $\mathrm{XX}$ & & & $\mathrm{X}$ & & $\mathrm{X}$ \\
\hline Vein & $\mathrm{X}$ & $\mathrm{X}$ & & & & & $\mathrm{X}$ \\
\hline Kidney & $\mathrm{XX}(\mathrm{S})$ & $\mathrm{XX}$ & $\mathrm{X}$ & & $\mathrm{X}$ & $\mathrm{X}$ & $\mathrm{X}$ \\
\hline Liver & $\mathrm{XX}(\mathrm{S})$ & $\mathrm{XX}(\mathrm{S})$ & $\mathrm{X}(\mathrm{S})$ & $\mathrm{X}$ & $\mathrm{X}(\mathrm{S})$ & & $\mathrm{X}$ \\
\hline Lung & $\mathrm{XX}$ & $\mathrm{XX}$ & & & $\mathrm{XX}$ & & $\mathrm{XX}$ \\
\hline Spleen & $\mathrm{XX}$ & $\mathrm{XX}$ & $\mathrm{X}$ & $\mathrm{XX}$ & & & $\mathrm{XX}$ \\
\hline Limb Muscle & $\mathrm{XX}$ & $\mathrm{XX}$ & & & & & $\mathrm{X}$ \\
\hline Pectoral M & $\mathrm{XX}$ & $\mathrm{XX}$ & & & & & $\mathrm{X}$ \\
\hline Adrenal & $\mathrm{XX}$ & $\mathrm{X}$ & & & $\mathrm{X}(\mathrm{S})$ & & $\mathrm{XX}$ \\
\hline Cartilage & $\mathrm{XX}$ & $\mathrm{X}$ & & & & & \\
\hline Bone & & & & & & & $\mathrm{X}$ \\
\hline Ovary & $\mathrm{XX}$ & $\mathrm{XX}$ & & & $\mathrm{X}$ & & $\mathrm{XX}$ \\
\hline Pancreas & $\mathrm{XX}$ & $\mathrm{X}$ & & & $\mathrm{X}$ & & $\mathrm{XX}$ \\
\hline Dermis & $\mathrm{XX}$ & $\mathrm{XX}$ & & & & & $\mathrm{X}$ \\
\hline S. Intestine & $\mathrm{XX}$ & & & & & & $\mathrm{X}$ \\
\hline Stomach & $\mathrm{XX}$ & $\mathrm{XX}$ & & $\mathrm{X}$ & $\mathrm{XX}$ & & \\
\hline
\end{tabular}




\section{References}

Amthor, H., Christ, B., and Patel, K. (1999). A molecular mechanism enabling continuous embryonic muscle growth - a balance between proliferation and differentiation. Development $126,1041-1053$.

Amthor, H., Christ, B., Weil, M., and Patel, K. (1998). The importance of timing differentiation during limb muscle development. Curr Biol 8, 642-652.

Bardot, B., Lecoin, L., Fliniaux, I., Huillard, E., Marx, M., and Viallet, J.P. (2004).

Drm/Gremlin, a BMP antagonist, defines the interbud region during feather development. Int J Dev Biol 48, 149-156.

Bernfield, M., Gotte, M., Park, P.W., Reizes, O., Fitzgerald, M.L., Lincecum, J., and Zako, M. (1999). Functions of cell surface heparan sulfate proteoglycans. Annu Rev Biochem 68, 729-777.

Bovolenta, P., Esteve, P., Ruiz, J.M., Cisneros, E., and Lopez-Rios, J. (2008). Beyond Wnt inhibition: new functions of secreted Frizzled-related proteins in development and disease. $\mathrm{J}$ Cell Sci 121, 737-746.

Capdevila, J., and Johnson, R.L. (2000). Hedgehog signaling in vertebrate and invertebrate limb patterning. Cell Mol Life Sci 57, 1682-1694.

Capurro, M.I., Xu, P., Shi, W., Li, F., Jia, A., and Filmus, J. (2008). Glypican-3 inhibits Hedgehog signaling during development by competing with patched for Hedgehog binding. Dev Cell 14, 700-711.

Chen, C.W., Jung, H.S., Jiang, T.X., and Chuong, C.M. (1997). Asymmetric expression of Notch/Delta/Serrate is associated with the anterior-posterior axis of feather buds. Dev Biol 188, 181-187.

Chiao, E., Fisher, P., Crisponi, L., Deiana, M., Dragatsis, I., Schlessinger, D., Pilia, G., and Efstratiadis, A. (2002). Overgrowth of a mouse model of the Simpson-Golabi-Behmel syndrome is independent of IGF signaling. Dev Biol 243, 185-206.

Dephoure, N., Gould, K.L., Gygi, S.P., and Kellogg, D.R. (2013). Mapping and analysis of phosphorylation sites: a quick guide for cell biologists. Mol Biol Cell 24, 535-542.

Desbiens, X., Turque, N., and Vandenbunder, B. (1992). Hydrocortisone perturbs the cell proliferation pattern during feather morphogenesis: evidence for disturbance of cephalocaudal orientation. Int J Dev Biol 36, 373-380.

Dwivedi, P.P., Grose, R.H., Filmus, J., Hii, C.S., Xian, C.J., Anderson, P.J., and Powell, B.C. (2013). Regulation of bone morphogenetic protein signalling and cranial osteogenesis by Gpc1 and Gpc3. Bone 55, 367-376.

Fico, A., Maina, F., and Dono, R. (2011). Fine-tuning of cell signaling by glypicans. Cell Mol Life Sci 68, 923-929.

Galli, A., Roure, A., Zeller, R., and Dono, R. (2003). Glypican 4 modulates FGF signalling and regulates dorsoventral forebrain patterning in Xenopus embryos. Development 130, 4919-4929.

Hamburger, V., and Hamilton, H.L. (1951). A series of normal stages in the development of the chick embryo. J Morphol 88, 49-92.

Kim, S.H., Turnbull, J., and Guimond, S. (2011). Extracellular matrix and cell signalling: the dynamic cooperation of integrin, proteoglycan and growth factor receptor. J Endocrinol 209, 139-151.

Kreuger, J., and Kjellen, L. (2012). Heparan sulfate biosynthesis: regulation and variability. J Histochem Cytochem 60, 898-907. 
Kreuger, J., Perez, L., Giraldez, A.J., and Cohen, S.M. (2004). Opposing activities of Dallylike glypican at high and low levels of Wingless morphogen activity. Dev Cell 7, 503-512. Lai, J.P., Sandhu, D.S., Yu, C., Han, T., Moser, C.D., Jackson, K.K., Guerrero, R.B., Aderca, I., Isomoto, H., Garrity-Park, M.M., et al. (2008). Sulfatase 2 up-regulates glypican 3, promotes fibroblast growth factor signaling, and decreases survival in hepatocellular carcinoma. Hepatology 47, 1211-1222.

McKinnell, I.W., Turmaine, M., and Patel, K. (2004). Sonic Hedgehog functions by localizing the region of proliferation in early developing feather buds. Dev Biol 272, 76-88. Nieto, M.A., Patel, K., and Wilkinson, D.G. (1996). In situ hybridization analysis of chick embryos in whole mount and tissue sections. Methods Cell Biol 51, 219-235.

Parr, B.A., and McMahon, A.P. (1995). Dorsalizing signal Wnt-7a required for normal polarity of D-V and A-P axes of mouse limb. Nature 374, 350-353.

Patel, K., Makarenkova, H., and Jung, H.S. (1999). The role of long range, local and direct signalling molecules during chick feather bud development involving the BMPs, follistatin and the Eph receptor tyrosine kinase Eph-A4. Mech Dev 86, 51-62.

Perriton, C.L., Powles, N., Chiang, C., Maconochie, M.K., and Cohn, M.J. (2002). Sonic hedgehog signaling from the urethral epithelium controls external genital development. Dev Biol 247, 26-46.

Petiot, A., Conti, F.J., Grose, R., Revest, J.M., Hodivala-Dilke, K.M., and Dickson, C. (2003). A crucial role for Fgfr2-IIIb signalling in epidermal development and hair follicle patterning. Development 130, 5493-5501.

Pilia, G., Hughes-Benzie, R.M., MacKenzie, A., Baybayan, P., Chen, E.Y., Huber, R., Neri, G., Cao, A., Forabosco, A., and Schlessinger, D. (1996). Mutations in GPC3, a glypican gene, cause the Simpson-Golabi-Behmel overgrowth syndrome. Nat Genet 12, 241-247. Qiao, D., Meyer, K., Mundhenke, C., Drew, S.A., and Friedl, A. (2003). Heparan sulfate proteoglycans as regulators of fibroblast growth factor-2 signaling in brain endothelial cells. Specific role for glypican-1 in glioma angiogenesis. J Biol Chem 278, 16045-16053. Rodriguez-Esteban, C., Tsukui, T., Yonei, S., Magallon, J., Tamura, K., and Izpisua Belmonte, J.C. (1999). The T-box genes Tbx4 and Tbx5 regulate limb outgrowth and identity. Nature 398, 814-818.

Sanz-Ezquerro, J.J., and Tickle, C. (2000). Autoregulation of Shh expression and Shh induction of cell death suggest a mechanism for modulating polarising activity during chick limb development. Development 127, 4811-4823.

Sarrazin, S., Lamanna, W.C., and Esko, J.D. (2011). Heparan sulfate proteoglycans. Cold Spring Harb Perspect Biol 3.

Smith, J. (1997). Brachyury and the T-box genes. Curr Opin Genet Dev 7, 474-480.

Tickle, C. (2004). The contribution of chicken embryology to the understanding of vertebrate limb development. Mech Dev 121, 1019-1029.

Towers, M., and Tickle, C. (2009). Generation of pattern and form in the developing limb. Int J Dev Biol 53, 805-812.

Ueki, Y., and Reh, T.A. (2012). Activation of BMP-Smad1/5/8 signaling promotes survival of retinal ganglion cells after damage in vivo. PLoS One 7, e38690.

Veugelers, M., De Cat, B., Ceulemans, H., Bruystens, A.M., Coomans, C., Durr, J., Vermeesch, J., Marynen, P., and David, G. (1999). Glypican-6, a new member of the glypican family of cell surface heparan sulfate proteoglycans. J Biol Chem 274, 2696826977.

Widelitz, R.B., and Chuong, C.M. (1999). Early events in skin appendage formation: induction of epithelial placodes and condensation of dermal mesenchyme. J Investig Dermatol Symp Proc 4, 302-306. 
Widelitz, R.B., Jiang, T.X., Chen, C.W., Stott, N.S., Jung, H.S., and Chuong, C.M. (1999). Wnt-7a in feather morphogenesis: involvement of anterior-posterior asymmetry and proximal-distal elongation demonstrated with an in vitro reconstitution model. Development 126, 2577-2587.

Wu, Y., Belenkaya, T.Y., and Lin, X. (2010). Dual roles of Drosophila glypican Dally-like in Wingless/Wnt signaling and distribution. Methods Enzymol 480, 33-50.

Yan, D., and Lin, X. (2009). Shaping morphogen gradients by proteoglycans. Cold Spring Harb Perspect Biol 1, a002493. 